\title{
Malin 1: A Deeper Look
}

\author{
Lesa Moore ${ }^{\mathrm{A}, \mathrm{C}}$ and Quentin A. Parker ${ }^{\mathrm{A}, \mathrm{B}}$ \\ A Department of Physics, Macquarie University, Sydney NSW 2109, Australia \\ B Anglo-Australian Observatory, PO Box 296, Epping NSW 2121, Australia \\ C Corresponding author. E-mail: lmoore@ics.mq.edu.au
}

Received 2006 September 22, accepted 2007 February 19

\begin{abstract}
Our $R$-band data show that the optical light from Malin 1 corresponds well with the $>2$-arcmin extent of the galaxy's Hi content and continues well beyond previously published $V$-band optical light radial profiles. Analysis of our image yields improved understanding of the galaxy's properties. We measure ellipticity of $0.20 \pm 0.03$, implying inclination of $38 \pm 3^{\circ}$, and we trace the radial profile to 77 arcsec. A single dusty spiral arm is also weakly discernable, and is consistent with the rotation direction of the Hi and spiral structure of the inner disk. Possible scenarios for the origin of the spiral structure are discussed.
\end{abstract}

Keywords: galaxies: evolution — galaxies: fundamental parameters — galaxies: individual (Malin 1) — techniques: image processing

\section{Introduction}

The knowledge of galaxy properties has always been constrained by limitations of the detector and background noise. By using star counts from deep CCD imaging, Bland-Hawthorn et al. (2005) traced stars in the outer stellar disk of NGC 300 to 10 scale lengths, doubling the previously known radial extent of the optical disk. There is still much to by learned be re-examining objects with improved methods. Here, traditional astrophotography combined with digital scanning and stacking enables the outer reaches of the enigmatic galaxy, Malin 1, to be studied to unprecedented depth. Previous image stacks have combined up to 13 films (Katsiyannis et al. 1998). Our image is derived from a set of 63 UK Schmidt Telescope (UKST) films providing a 2.25-mag gain over the depth of a single film (Bland-Hawthorn, Shopbell, \& Malin 1993). The image is centred near M87 (the northern concentration of Virgo Cluster galaxies) and covers an unvignetted field of 25 square degrees. The background galaxy, Malin 1, also lies within this field.

Malin 1 is a highly unusual galaxy that remains the most extreme in the class of low surface brightness (LSB) giants (Pickering et al. 1997). A recent study by Barth (2007) suggests the galaxy has an inner normal barred spiral disk (SB0/a) surrounded by a large diffuse LSB envelope. Nevertheless our data show this galaxy's outer disk extends to around $120 \mathrm{kpc}$ making it the largest diffuse outer region of any known disk galaxy. It has a large $M_{\mathrm{HI}} / L_{\mathrm{B}}$ ratio (Impey \& Bothun 1989) and appears to be an isolated galaxy that has evolved slowly. To date, analyses of the properties of Malin 1 have been hampered by the fact that scaling of the Hi rotation curve, and derivation of dependent quantities, rely on knowing the inclination of the galaxy (Pickering et al. 1997). The best measurements from CCD images have large uncertainty in the inclination (e.g. Pickering et al. (1997) quote inclination of $45 \pm 15^{\circ}$ ). Improved measurements from our deeper image allow us to refine this measurement and reduce the uncertainty considerably.

A summary of previously published data on Malin 1 is included in Section 2. Our data and analysis are described in Section 3. In Section 4 we consider the significance of our new findings.

\section{Existing Data}

The discovery of Malin 1 by Bothun et al. (1987) was made by studying a photographically amplified image. These authors followed up with deep $V$-band CCD imaging to derive basic optical parameters for the galaxy, and Hi observations with the Arecibo radio telescope to measure redshift. Malin 1 is acknowledged as being both massive and dark matter dominated (Pickering et al. 1997). The published radial profile of Bothun et al. (1987) extends to $\sim 65$ arcsec in $V$ band. Their fit to the disk yields an extrapolated central surface brightness of $V(0)=25.7 \pm 0.1 \mathrm{mag} \operatorname{arcsec}^{-2}$ and disk scale length of $45 \pm 5$ arcsec. Table 1 contains summary results from Bothun et al. (1987) and Pickering et al. (1997). Distance-dependent quantities are based on the Bothun et al. (1987) $\mathrm{HI}$ redshift, and assume $H_{0}=$ $75 \mathrm{~km} \mathrm{~s}^{-1} \mathrm{Mpc}^{-1}$ and a derived distance of $330 \mathrm{Mpc} h_{75}^{-1}$. Values in the table are those of Pickering et al. (1997) unless otherwise indicated.

Measurements of Hi mass range between the Impey \& Bothun (1989) value of $6.7 \times 10^{10} \mathrm{M}_{\odot}$ (for $H_{0}=$ $100 \mathrm{~km} \mathrm{~s}^{-1} \mathrm{Mpc}^{-1}$ ) to that of Radford (1992), $1.96 \times 10^{11} \mathrm{M}_{\odot}$ (for $H_{0}=75 \mathrm{~km} \mathrm{~s}^{-1} \mathrm{Mpc}^{-1}$ ). Certainly 
Table 1. Published data on Malin 1

\begin{tabular}{lc}
\hline Property & Details $^{\mathrm{A}}$ \\
\hline RA (1950) & $12^{\mathrm{h}} 34^{\mathrm{m}} 28^{\mathrm{s}} .1$ \\
Dec (1950) & $+14^{\circ} 36^{\prime} 18^{\prime \prime} .1$ \\
Redshift & $0.083^{\mathrm{B}}$ \\
Hi velocity & $2475 \pm \pm 10 \mathrm{~km} \mathrm{~s}^{-1}$ \\
Scale length & $45 \pm 5^{\prime \prime} \mathrm{B}^{-1}$ \\
Quoted scale size & $82 \mathrm{kpc} h_{75}^{-1}$ \\
Optical radius & $65 \operatorname{arcsec}^{\mathrm{B}}$ \\
Inclination & $45 \pm 15^{\circ}$ \\
Hi diameter & up to $3.3 \operatorname{arcmin}^{\mathrm{C}}$ \\
\hline
\end{tabular}

A Pickering et al. (1997) unless otherwise stated.

B Bothun et al. (1987).

${ }^{\mathrm{C}}$ Impey \& Bothun (1989).

the $\mathrm{HI}$ mass is significant and the $M_{\mathrm{HI}} / L_{\mathrm{B}}$ ratio is also large at $\sim 3$ (Impey \& Bothun 1989). Matthews, van Driel, \& Monnier-Ragaigne (2001) highlight that additional surface photometry and Hi measurements are needed to improve our measurements and understanding of the global properties of giant LSB galaxies.

\section{Malin 1 Image Data and Analysis}

The image of Malin 1 (base image of Figures 1, 2 and 3) is derived from 63 UKST films that have been digitally scanned by SuperCOSMOS and co-added to produce a unique ultra-deep $R$-band image covering 36 square degrees. The 63 A-grade Tech-Pan films were taken with an OG590 filter between January and June in 1999, 2000 and 2001. Exposure times were mostly 60.0 minutes, though 13 were a little shorter, probably due to interference by cloud. The field centre is at $12^{\mathrm{h}} 27^{\mathrm{m}} 00^{\mathrm{s}}$ $+13^{\circ} 30^{\prime \prime} 00^{\prime}$ (B1950). This places Malin 1 in the upper left portion of the UKST field, though not so close to the edge as to be affected by vignetting. The range in data counts is optimised for the faint end in the scanning process, so bright stars and galaxy cores are affected by saturation.

The stacking of films increases signal to noise, having the effect of pushing down the background to reveal fainter parts of the galaxy than are visible in a single film.

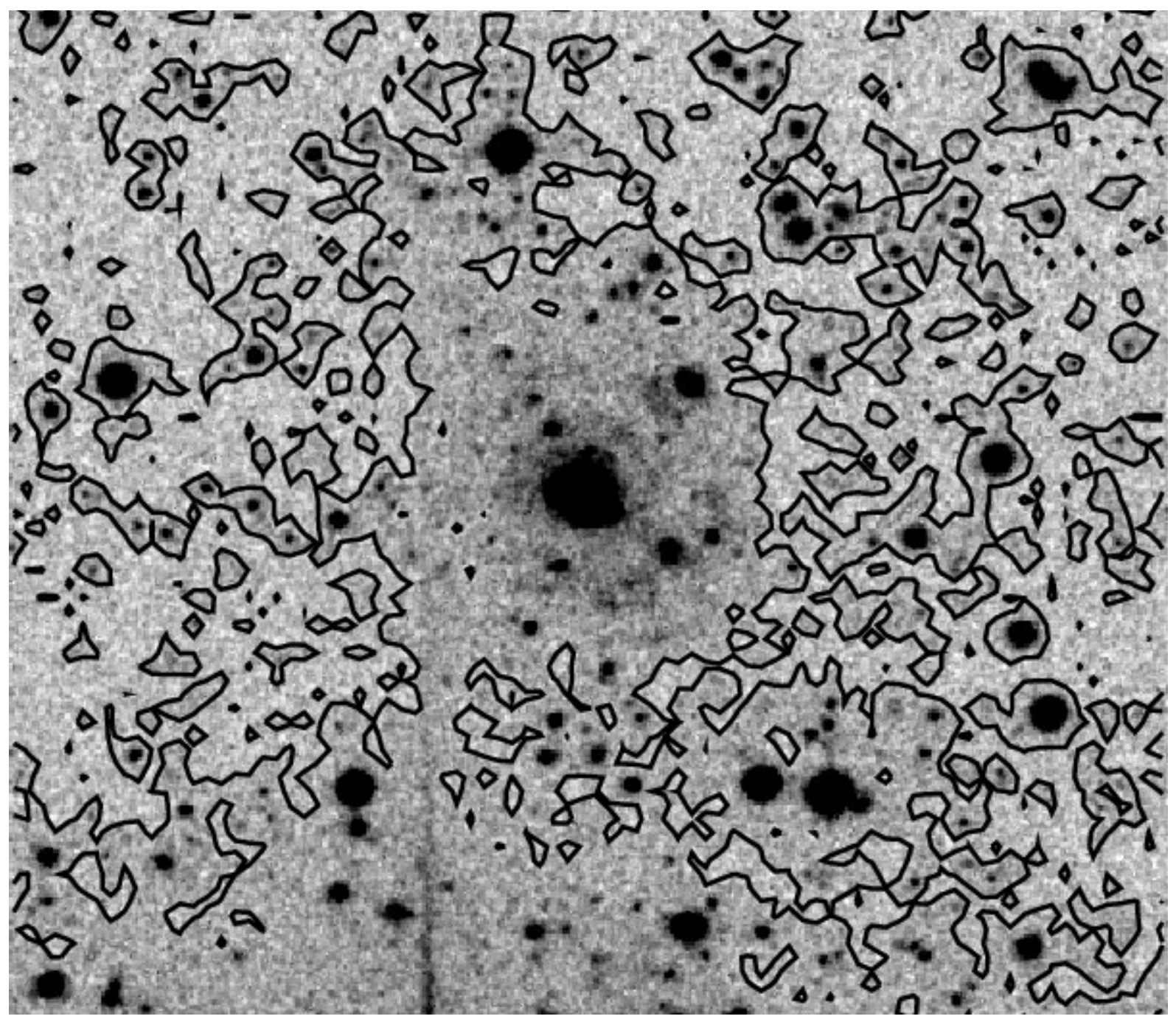

Figure 1 Malin 1 with contours at 6380 data counts to delineate the extent of the optical disk. This image measures 5'36" on a side. The vertical feature (lower left) is a diffraction spike from a star off the bottom edge of the image. North is at the top, east is to the left. 


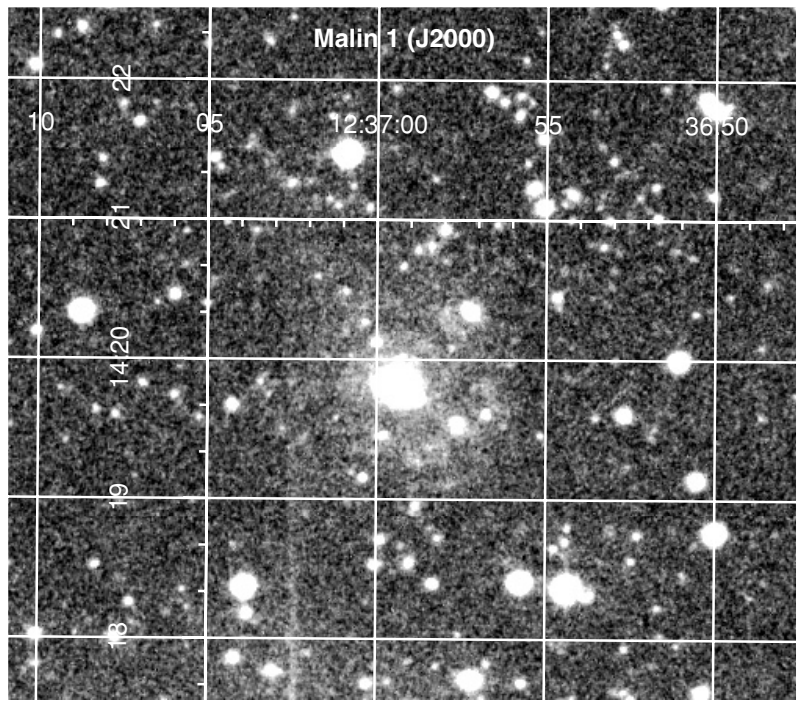

Figure 2 Image of Malin 1 from the 63-film stack. Coordinate grid is $\mathbf{J} 2000$.

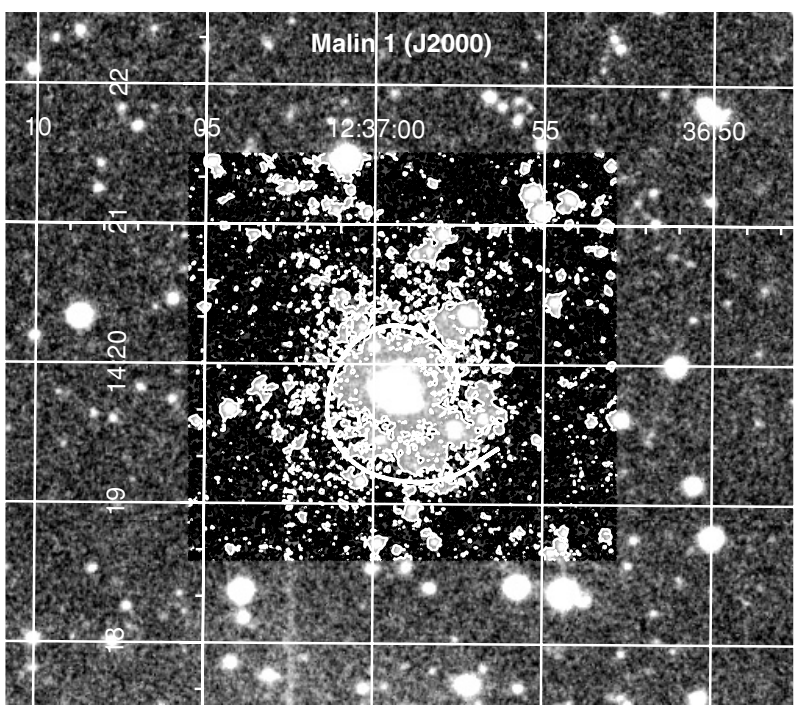

Figure 3 Malin 1 with dark and light contours overlaid to enhance intensity variations in the image. The drawn line traces the position of the dusty spiral arm detected in the image.

The magnitude gain from stacking $N$ films is given by Bland-Hawthorn, Shopbell, \& Malin (1993) as $2.5 \mathrm{log}$ $\sqrt{ } N$ magnitudes. Hence, the deep-stack image goes 2.25 magnitudes deeper than a single Tech-Pan film, which is already one magnitude deeper than traditional IIIa emulsions used on glass plates (Parker \& Malin 1999).

Calibration of the image was performed by B. J. Jones (2006, private communication) by selecting 20 stars in the magnitude range $18.2 \leq$ Cousins $R \leq 20.1$ from the Sloan Digital Sky Survey DR4 online catalogue and using aperture photometry on the same stars in the image. A best fit to the magnitudes yields a calibration constant that can then be applied to the conversion between data counts and magnitude anywhere on the image. The calibration constant thus found is 31.54 .

\section{Malin $1 \boldsymbol{R}$-band radial profile}

Arcsec

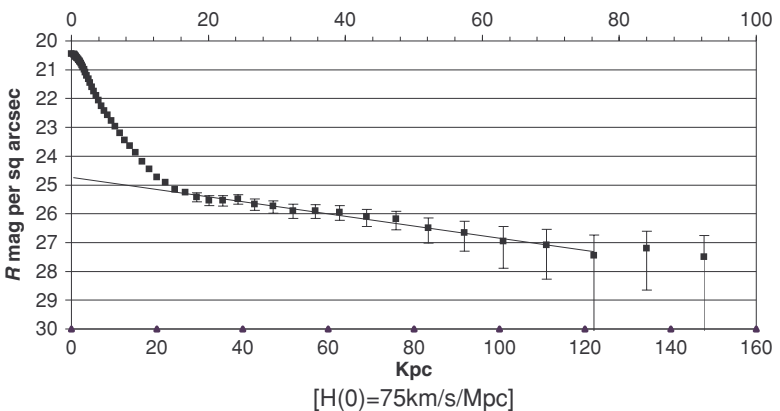

Residuals

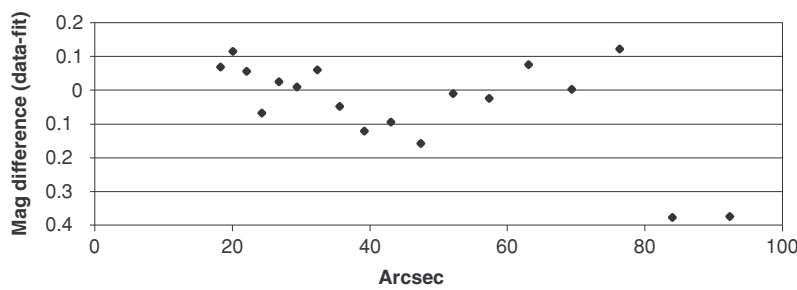

Figure 4 Top: The $R$-band radial profile shows that the optical disk (solid squares) is visible to a semi-major axis of $\sim 80$ arcsec before reaching the background level. The upper scale shows arcsec. The lower scale shows kpc based on the redshift given in Table 1 and assuming $H_{0}=75 \mathrm{~km} \mathrm{~s}^{-1} \mathrm{Mpc}^{-1}$. A fit to the exponential part of the profile is shown as a solid line. The last two data points on the right are not included in the fit as these are clearly beyond the point where the galaxy light can be distinguished from sky and adjacent objects. The fit is extrapolated to the left to show central surface brightness of $24.74 R$ mag $\operatorname{arcsec}^{-2}$. See text for explanation of the calculation of the error bars on the exponential part of the profile. Bottom: Residuals from the fit (data minus fit), also showing the variance of the last two data points from the fit.

Our data show that the $R$-band optical light from Malin 1 continues even further than the Hi mapped by Pickering et al. (1997) and reproduced by Braine, Herpin, \& Radford (2000). Contours at 6380 data counts on the image are displayed in Figure 1 and trace the irregular outer extent of the galaxy. We also find the radial profile (Figure 4) extends to almost 80 arcsec, well beyond the $V$-band profiles of Bothun et al. (1987) and Pickering et al. (1997). The Bothun et al. (1987) profile only extends to $\sim 65$ arcsec, and the Pickering et al. (1997) profile is plotted to $\sim 80 \mathrm{kpc}$, equating to approximately 50 arcsec (where they have used $H_{0}=75 \mathrm{~km} \mathrm{~s}^{-1} \mathrm{Mpc}^{-1}$ ).

Ellipse parameters of the outer isophotes of Malin 1 were determined by matching ellipses by eye to contours overlaid on the image at intervals of 20 data counts in the range 6360 to 6460 inclusive. These were displayed in DS9 and ellipse dimensions and position angles read off. The mean values thus derived are ellipticity $e=$ $0.20 \pm 0.03(e=1-b / a$ where $a$ and $b$ are major and minor axes respectively), and position angle $\phi=43 \pm 3^{\circ}$. The former equates to an inclination of $38 \pm 3^{\circ}$. 
Combinations of these parameters were used as fixed values for surface photometry in the IRAF task ELLIPSE with resulting profiles yielding only small variations in extrapolated central surface brightness and scale length.

The radial profile from the surface photometry using ellipticity of 0.20 and position angle of 40 degrees is shown in Figure 4. Note that the outer parts of the radial profile are highly sensitive to the background value used. The sky background level was measured in five carefully selected separate regions from 2.5 to 3 arcmin away from the galaxy's optical centre. These elliptical regions, arranged on three sides of the galaxy and each covering $\sim 2100$ pixels, were evaluated in GAIA to obtain separate mean and standard deviation for each. The five results were then averaged to arrive at a final mean of 6364 and standard deviation of 38 data counts.

The radial profile (Figure 4) may be traced to 77 arcsec on the semi-major axis of the outermost fitted ellipse before levelling out to the sky background. This is equivalent to $2.3 \pm 0.1$ scale lengths or $124 \mathrm{kpc} h_{75}^{-1}$. This does not include an extension to the north-east, which does appear from our image to be contiguous with Malin 1, but could be a tidal tail, an interacting or completely separate object.

The largest source of error in the outer part of the profile is due to uncertainty in the sky background measurement. Ideally the errors on the data points in the profile should be derived by summing in quadrature the errors in signal and sky. Unfortunately, this fails when attempting to convert a resulting negative value (where signal minus error is less than sky) to magnitude. Instead, errors here are derived from recalculations of magnitudes using: $s k y_{\text {high/low }}=s k y_{\text {mean }} \pm\left(0.5 \sigma_{\text {sky }}\right)$.

The fit to the exponential part of the radial profile yields a value of $24.74(+0.07 /-0.2) R$ mag $\operatorname{arcsec}^{-2}$ for extrapolated central surface brightness, with errors taken from extrapolated fits to error bars. Scale length of the fit is measured at $33 \operatorname{arcsec}\left(53 \mathrm{kpc} h_{75}^{-1}\right)$, which is smaller than the values obtained by either Bothun et al. (1987) or Pickering et al. (1997). We also find a variation in position angle of fitted ellipses that may support the warping mentioned by Pickering et al. (1997).

Inspection of the image at high contrast levels (Figure 2) and enhancement using overlaid contours reveal for the first time the likely presence of a single dusty spiral arm. Barth (2007) comments that spiral structure is often seen in outer disks of early-type barred galaxies and he detects a hint of spiral structure in his HST I-band image of Malin 1. Clearly, it is difficult to discern such structure at this magnitude where contrast with the background is so low. For the reader's benefit, contours and the position of the spiral arm are drawn onto Figure 3. Inspection of a highresolution jpeg version of David Malin's $B$-band image (http://www.aao.gov.au/images/deep_html/malin-1_d.html) shows tonal variations consistent with our measurements.

\section{Discussion}

The apparent presence of a single dusty spiral arm has some profound implications. Asymmetric spiral forma- tion may be a natural consequence of the lack of a gaseous damping process (Phookun, Vogel, \& Mundy 1993), which stands to reason when there is low gas surface density. The shape of the spiral arm is consistent with the rotation direction of the Hi mapped by Pickering et al. (1997) and the detailed view of the inner disk studied by Barth (2007). Modelling of the formation of spiral arm structure is beyond the scope of this paper, but our detection poses the question: Could a single spiral arm evolve naturally in a giant quiescent disk galaxy?

In a previous study of a one-armed spiral galaxy Phookun, Vogel, \& Mundy (1993) imply that this type of structure is more likely the result of an interaction with an infalling gas cloud. Modelling by Peñarrubia, McConnachie, \& Babul (2006) shows that a diffuse outer disk could result from tidal shredding of a dwarf galaxy. If this occurred and was relatively recent, it could account for the observed asymmetry. However, theories on the nature of LSB galaxies suggest that star formation is inhibited by low gas surface density (Kennicutt 1989) and a quiescent history without interactions. Therefore it seems that if the latter idea is correct, the single-arm structure should not be due to an interaction.

On the other hand, from our image we also discern a faint optical lobe to the north-east (near top, left of centre in Figure 1). Whilst it is impossible to say, without further detailed observation, whether or not this is associated with Malin 1, we conjecture that this may be part of a system undergoing 'quiescent merger'. This may explain both the single-arm structure, as material is gently accreting onto the galaxy, and the observed low surface brightness, maintained through lack of shocks that might otherwise trigger star formation. We note that the optical light we detect for the galaxy itself corresponds directly with the HI map of Pickering et al. (1997), extending across more than 2 arcmin. Though the Hi mapped by Pickering et al. (1997) does not appear to extend into the north-east lobe region, their data show some extension in that direction.

If the galaxy is undergoing a quiescent merger, it is likely that this process is extremely rare and may depend on the unique properties of Malin 1, i.e. its $M_{\mathrm{HI}} / L_{\mathrm{B}}$ of 3 (Impey \& Bothun 1989), its high dark matter content (Pickering et al. 1997) and isolation from neighbouring galaxies.

\section{Acknowledgements}

We wish to thank Bryn Jones for his calibration of the image stack and his helpful private communications. We also thank David Malin for the opportunity to study his $B$-band image of Malin 1 .

\section{References}

Barth, A. J. 2007, AJ, 133, 1085

Bland-Hawthorn, J., Shopbell, P. L. \& Malin, D. F. 1993, AJ, 106, 2154

Bland-Hawthorn, J., Vlajić, M., Freeman, K. C. \& Draine, B. T. 2005, ApJ, 629, 239 
Bothun, G. D., Impey, C. D., Malin, D. F. \& Mould, J. R. 1987, AJ, 94, 23

Braine, J., Herpin, F. \& Radford, S. J. E. 2000, A\&A, 358, 494

Impey, C. \& Bothun, G. 1989, ApJ, 341, 89

Katsiyannis, A. C., Kemp, S. N., Berry, D. S. \& Meaburn, J. 1998, A\&AS, 132, 387

Kennicutt, R. C.Jr., AJ, 344, 685
Matthews, L. D., van Driel, W. \& Monnier-Ragaigne, D. 2001, A\&A, 365, 1

Parker, Q. A. \& Malin, D. 1999, PASA, 16, 288

Peñarrubia, J., McConnachie, A. \& Babul, A. 2006, ApJ, 650, L33

Phookun, B., Vogel, S. N. \& Mundy, L. G. 1993, ApJ, 418, 113

Pickering, T. E., Impey, C. D., van Gorkom, J. H. \& Bothun, G. D. 1997, AJ, 114, 1858

Radford, S. J. E. 1992, A\&A, 262, 13 